\title{
Associations among Physical Activity, Comorbidity, Functional Capacity, Peripheral Muscle Strength and Depression in Breast Cancer Survivors
}

\author{
Naciye Vardar-Yagli ${ }^{1 *}$, Gul Sener ${ }^{1}$, Melda Saglam ${ }^{1}$, Ebru Calik-Kutukcu ${ }^{1}$, Hulya \\ Arikan $^{1}$, Deniz Inal-Ince ${ }^{1}$, Sema Savci², Kadri Altundag ${ }^{3}$, Tezer Kutluk ${ }^{4}$, Yavuz \\ Ozısık $^{3}$, Ergun Barıs Kaya ${ }^{5}$
}

\begin{abstract}
Physical inactivity may an important outcome in the prognosis of breast cancer. Physical activity levels decrease significantly for breast cancer patients following the treatment and remain low after oncology treatment is completed. The aim of this study was to determine physical activity levels and to examine associations among physical activity, comorbidity, functional capacity, peripheral muscle strength, and psychosocial status in breast cancer patients. Forty breast cancer survivors were included in this study. Demographic and clinical characteristics were recorded. Physical activity was assessed with a short form of the International Physical Activity Questionnaire (IPAQ). We defined comorbid diseases according to the Charlson Comorbidity Index (CCI). Functional capacity was evaluated with a six-minute walk test (6MWT). Peripheral muscle strength was measured for quadriceps femoris muscle with a hand-held dynamometer. Psychosocial status was measured with the Hospital Anxiety Depression Scale (HADS). Forty percent of patients were inactive. The IPAQ total score was significantly related with quadriceps muscle strength $(r=0.492 ; p<0.001)$ and HADS depression score $(\mathrm{r}=0.341 ; \mathbf{p}<0.05)$. Marked correlations were also observed between IPAQ walking score and quadriceps muscle strength $(r=0.449 ; p<0.001)$, HADS depression score $(r=0.341 ; p<0.05)$, and CCI $(r=-0.433 ; p<0.001)$. The CCI score was also markedly associated with quadriceps muscle strength $(r=-0.413 ; p<0.001)$. 6MWT distance was not significantly correlated with any of the parameters. Regression analyses revealed that psychosocial status and peripheral muscle strength were significant predictors of physical activity estimated with the IPAQ short form and when combined, they explained $35 \%$ of the variance. Comorbidities, peripheral muscle strength and psychosocial status partially explain the variability of physical activity level in breast cancer survivors. These results suggest that physical inactivity contributes to worsening health in breast cancer survivors.
\end{abstract}

Keywords: Physical activity - breast cancer - muscle strength - comorbidity - depression

Asian Pac J Cancer Prev, 16 (2), 585-589

\section{Introduction}

Physical inactivity is a seriously growing problem and public health priority among individuals worldwide. Physical inactivity contributes to the risk of breast cancer, one of the most prevalent cancer in the world (Parkin, 2011), even an increase is expected in next years of the current burden of cancer associated with physical inactivity (Wang et al., 2012; Ariza-Garcia et al., 2013).

Recent literature have demonstrated that regular physical activity reduced the risk of breast cancer related morbidity and mortality as well as controlling side effects and disease progression, reducing psychological conditions (Burnham and Wilcox, 2002; McTiernan, 2008; Galanti et al., 2013).
However, despite all the evidence, most of the breast cancer survivors remain physically inactive. Motivating the breast cancer patients to engage in regular physical activity may be an enormous challenge due to side effects especially fatigue after chemotherapy and other treatments (Jacobsen et al., 1999). Some of the common side effects of breast cancer treatment that effected the level of physical activity include fatigue, muscle strength weakness, reduced aerobic exercise capacity, depression, bone mineral loss, obesity, and finally, impaired quality of life (Loprinzi and Cardinal, 2012).

Most of the breast cancer patients suffer psychological problems (Bleiker et al., 2000; Zainal et al., 2013; AbuHelalah et al., 2014) because of pain and associated functional limitations after treatment (Cantarero-

${ }^{1}$ Department of Physiotherapy and Rehabilitation, Faculty of Health Sciences, ${ }^{3}$ Department of Medical Oncology, ${ }^{4}$ Department of Pediatric Oncology, ${ }^{5}$ Department of Cardiology, Faculty of Medicine, Hacettepe University, Ankara ${ }^{2}$ School of Physiotherapy and Rehabilitation, Dokuz Eylul University, İzmir, Turkey*For correspondence: naciyevardar@yahoo.com 
Naciye Vardar-Yagli et al

Villanueva et al., 2011; Charlier et al., 2012). Studies demonstrated that eating practices physical activity related to pain and depressive mood (Sabiston et al., 2012; Mohammadi et al., 2013a;2013b).

In the current study, the aims were to evaluate the level of physical activity and to examine the associated factors as comorbidity, functional exercise capacity, peripheral muscle strength and psychosocial status in breast cancer survivors.

\section{Materials and Methods}

\section{Participants}

The study is carried out in the Cardiopulmonary Rehabilitation Unit of Physiotherapy and Rehabilitation Department. The physical and sociodemographic characteristics of all subjects were recorded. Forty patients aged between 20 and 60 years included who had received oncological treatment for stage I-II cancer, except hormone therapy. The patients who are mentally intact, and cancer treatment was completed at least three years ago participated in the study. The study was approved by the Ethics Committee of the University, and performed in accordance with the Declaration of Helsinki. Written informed consent was obtained from all the patients.

\section{Physical activity}

Physical activity was evaluated with short form of International Physical Activity Questionnaire (IPAQ). The IPAQ short form is a seven-item measure of four domains of activity: vigorous-intensity physical activity; moderate-intensity physical activity; walking and sitting. Participants reported frequency (during the last seven days) and duration of physical activity (minutes/hours usually spent on one of those days). Participants also report the total time they spend sitting on a week day during the last seven days. The participants were then categorized as inactive, minimally active and sufficiently active according to the IPAQ categorical classification (Saglam et al., 2010).

\section{Peripheral muscle strength}

Quadriceps femoris isometric strength was measured in the sitting position from non-affected side, using a handheld dynamometer (JTECH Power Track Commander II, USA). Quadriceps femoris muscle strength testing was repeated three times and the best value in Newtons $(\mathrm{N})$ was recorded (Bohannon, 2001).

\section{Functional capacity}

Functional exercise capacity was evaluated with six minute walk test (6MWT). The patients were requested to walk along a 30-meters flat corridor as fast as they could in their own walking speed for six minutes. Heart rate was recorded using heart rate monitoring (PE3000 Polar Electro, Finland) before and after the test. Pre and post-test general and quadriceps fatigue, and dyspnea perception were assessed with 10-point Modified Borg Scale (American Thoracic Society 2002).

\section{Psychosocial status}

Psychosocial status evaluated with Hospital Anxiety and Depression Scale (HADS). The HADS is screening the emotional disorder to measure anxiety (HADS-A) and depression (HADS-D) during the past week. Total subscale scores range from 0 to 21 . Cut off points for HADS-A score is 9 or more, and HADS-D score is 8 or more in breast cancer patients (Bjelland et al., 2002). The reliability and validity of the HADS subscales have been

\section{Table 1. Characteristics of the Patients}

\begin{tabular}{lc}
\hline Variables & Mean \pm SD \\
\hline Age, years & $48.58 \pm 6.41$ \\
Height, cm & $160.25 \pm 8.89$ \\
Weight, kg & $74.40 \pm 11.47$ \\
BMI, kg/m² & $29.22 \pm 5.76$ \\
Duration of disease, years & $4.58 \pm 1.62$ \\
6MWT distance, m & $510.65 \pm 44.60$ \\
6MWT distance, $\%$ & $92.90 \pm 9.85$ \\
Maximum heart rate, \% & $76.31 \pm 5.32$ \\
$\Delta D y s p n e a$, Borg & $2.51 \pm 1.81$ \\
$\Delta$ Fatigue, Borg & $3.43 \pm 1.60$ \\
$\Delta$ Leg Fatigue, Borg & $2.34 \pm 2.02$ \\
Charlson comorbidity severity & $2.00 \pm 0.91$ \\
IPAQ total PA, MET-min/wk & $1121.00 \pm 1143.75$ \\
IPAQ vigorous PA, MET-min/wk & $14.00 \pm 76.62$ \\
IPAQ moderate PA, MET-min/wk & $407.00 \pm 666.08$ \\
IPAQ walking, MET-min/wk & $699.75 \pm 768.76$ \\
IPAQ sitting score, min & $517.75 \pm 223.50$ \\
Quadriceps muscle strength, N & $312.65 \pm 83.85$ \\
HADS-anxiety (0-21) & $7.55 \pm 1.96$ \\
HADS-depression (0-21) & $5.08 \pm 1.72$ \\
HADS-total score & $12.63 \pm 2.61$ \\
\hline
\end{tabular}

*Values expressed as mean \pm SD. BMI, body mass index, HADS, Hospital Anxiety and Depression Score, IPAQ, International Physical Activity Questionnaire, 6MWT, six minute walk test, N, Newton

Table 2. Relationship between Functional Capacity, Physical Activity, Peripheral Muscle Strength, and Psychosocial Status

\begin{tabular}{|c|c|c|c|c|c|c|}
\hline & $\begin{array}{l}\text { IPAQ } \\
\text { total }\end{array}$ & $\begin{array}{c}\text { IPAQ } \\
\text { walking }\end{array}$ & $\begin{array}{l}\text { Quadriceps } \\
\text { strength }\end{array}$ & $\begin{array}{c}\text { 6MWT } \\
\text { distance }\end{array}$ & $\begin{array}{c}\text { HADS } \\
\text { depression }\end{array}$ & $\begin{array}{c}\text { Charlson } \\
\text { comorbidity }\end{array}$ \\
\hline IPAQ total & - & $0.824 * *$ & $0.492 * *$ & 0.087 & $0.341 *$ & -0.273 \\
\hline IPAQ walking & $0.824 * *$ & - & $0.449 * *$ & 0.117 & $0.341 *$ & $-0.413 * *$ \\
\hline Quadriceps & $0.492 * *$ & $0.449 * *$ & - & 0.168 & 0.109 & $-0.433 * *$ \\
\hline \multicolumn{7}{|l|}{ Strength } \\
\hline 6MWT distance & 0.087 & 0.117 & 0.168 & - & -0.123 & -0.194 \\
\hline HADS depression & $0.341 *$ & $0.341 *$ & 0.109 & -0.123 & - & 0.017 \\
\hline Charlson comorbidity & -0.273 & $-0.413 * *$ & $0.433 * *$ & -0.194 & 0.017 & - \\
\hline
\end{tabular}

*r: Pearson correlation coefficient, HADS, Hospital Anxiety and Depression Score, IPAQ, International Physical Activity Questionnaire, 6MWT, six minute walk test, $* * \mathrm{p}<0.001, * \mathrm{p}<0.05$ 
extensively established in oncology patients (Bjelland et al., 2002; Turhal et al., 2010).

\section{Comorbidity severity}

Comorbidity severity was evaluated with the Charlson Comorbidity Index, scores ranging from 0-37. Higher scores indicate worsening comorbidity (Braithwaite et al., 2012).

\section{Statistical analysis}

The statistical evaluation was performed using the SPSS 18.0 (SPSS Inc., Chicago, IL, USA) statistical packet software for Windows. Variables were descriptively expressed as mean \pm standard deviation (SD and frequency (\% values). Correlations between physical activity, comorbidity, and quadriceps femoris muscle strength, functional exercise capacity, and psychosocial status in patients with breast cancer were evaluated using the twotailed Pearson's correlation analysis. A forward stepwise multiple regression analysis evaluated how variables predicted physical activity levels. Variables with $\mathrm{p}<0.015$ were included as predictor variables for regression analysis. Significance was set at $\alpha<0.05$.

\section{Results}

Table 1 has shown the characteristics of the patient. Our sample consisted of 40 breast cancer patients with a mean age of $48.58 \pm 6.41$ years.
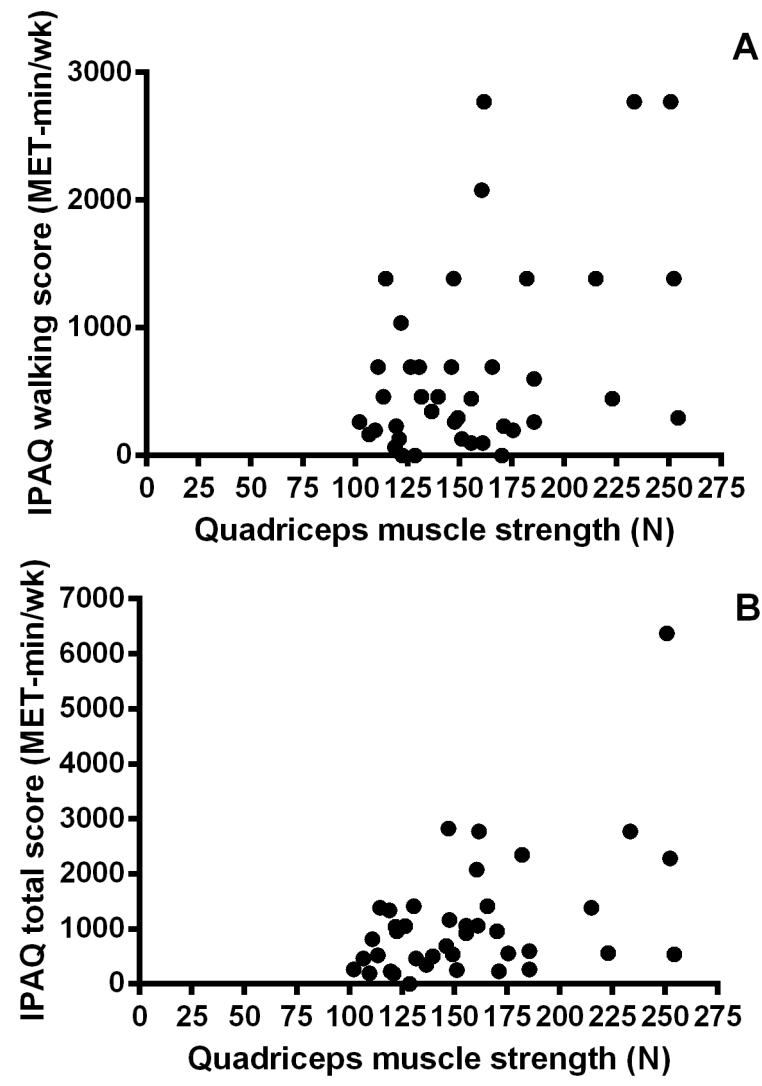

Figure 1. The Relationship between Walking Energy Expenditure with Peripheral Muscle Strength and Psychosocial Status. A) IPAQ walking score and quadriceps femoris muscle strength. B) IPAQ walking score and HADS depression score
According to IPAQ categorical classification, 16 (40\%) patients were inactive, $23(57.5 \%)$ patients were minimally active, and one $(2.5 \%)$ patient was sufficiently active in our study.

A marked correlation were observed between IPAQ walking score and quadriceps femoris muscle strength $(r=0.449 ; p<0.001$, Figure 1A); HADS-D score $(r=0.341$; $\mathrm{p}<0.05$, Figure $1 \mathrm{~B}$ ); and Charlson comorbidity index $(\mathrm{r}=-0.433 ; \mathrm{p}<0.001)$. Correlation analysis showed that IPAQ total score was significantly related with quadriceps muscle strength $(\mathrm{r}=0.492 ; \mathrm{p}<0.001$, Figure $2 \mathrm{~A})$ and HADS-D score $(r=0.341 ; p<0.05$, Figure $2 B)$. Charlson comorbidity index score was also obviously associated with quadriceps femoris muscle strength $(r=-0.413$; $\mathrm{p}<0.001)$. 6MWT distance was not significantly correlated with any of the parameters.
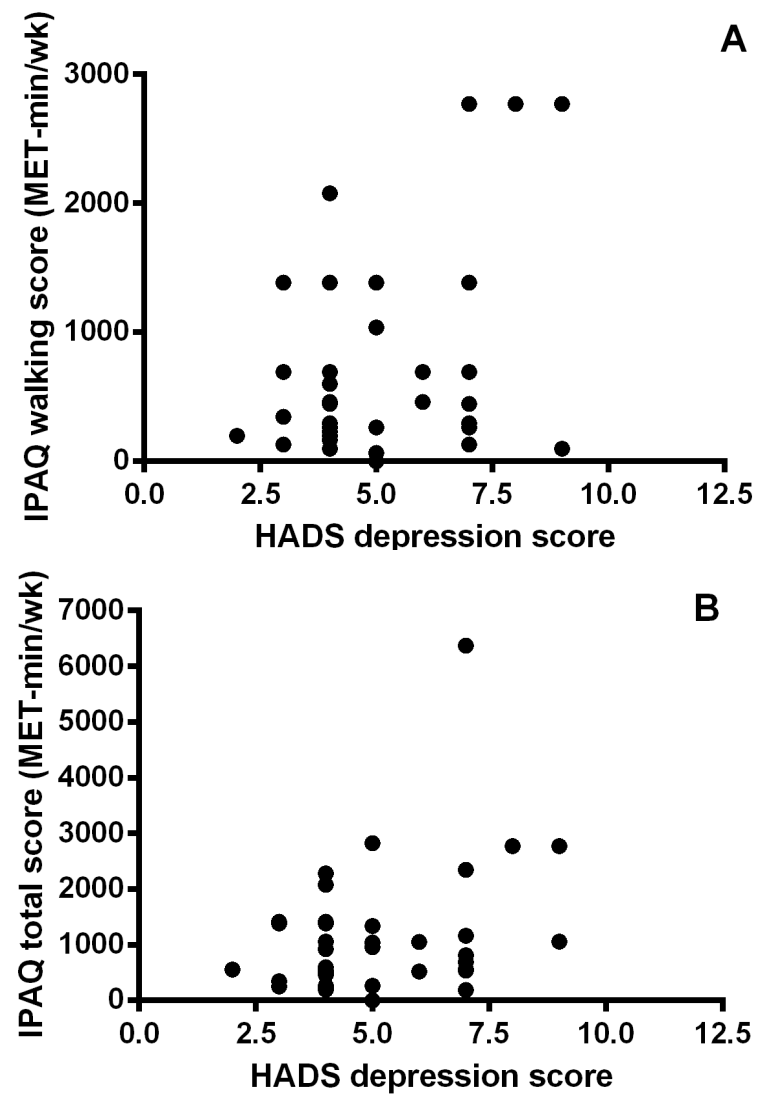

Figure 2. The Relationship between total Energy Expenditure with Peripheral Muscle Strength and Psychosocial Status A) IPAQ total score and quadriceps femoris muscle strength. B) IPAQ total score and HADS depression score

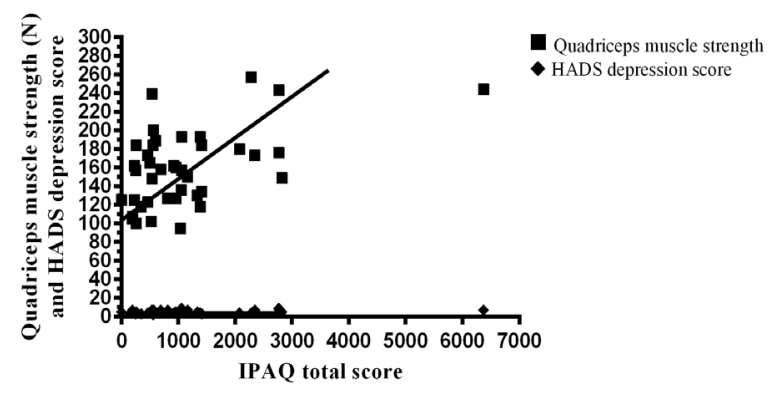

Figure 3. Physical Activity-Peripheral Muscle Strength and Psychosocial Status Regression Graphic 
Regression analyses revealed that psychosocial status and peripheral muscle strength were significant predictors of physical activity level that estimated with IPAQ short form and when combined, they explained $35 \%$ of the variance in physical activity level $\left(\mathrm{r}^{2}=0.354 ; \mathrm{F}=13.38\right.$; $\mathrm{p}<0.001$, Figure 3).

\section{Discussion}

To determine the physical activity level and to clarify the relationships between physical activity, comorbidity, peripheral muscle strength, functional capacity, and psychosocial status were the objectives of this study. The breast cancer patient sample in our study was not sufficiently active for health enhancing. We found significant moderate positive association between physical activity level and peripheral muscle strength, and moderate negative associations between physical activity level, comorbidity severity and psychosocial status. This finding showed that peripheral muscle strength and psychosocial status are important factors determining physical activity level.

Physical activity is a major component of the sufficient lifestyle in cancer survivors (Schmitz et al., 2010). Several studies on physical activity showed significant protective effect of physical activity on breast cancer risk (GalianoCastillo et al., 2013).

A part of non-communicable disease burden in the world, physical inactivity was also investigated in breast cancer. The prevalence of physical inactivity in the breast cancer population was found to be $38.8 \%$ in this study (Lee et al., 2012). This prevalence was similar to our study as our data showed that $40 \%$ of breast cancer patients have a sedentary lifestyle. This results support lack of exercise and increasing body weight in the breast cancer. In a meta-analysis evaluating 73 studies on physical activity and breast cancer demonstrated that increasing physical activity level reduced the risk of breast cancer by $25 \%$ (Courneya and Friedenreich, 2011; Lynch et al., 2011).

Comorbidities in breast cancer frequently include hypertension, gastrointestinal problems, diabetes mellitus, chronic bronchitis/asthma, coronary heart disease, and stroke. Physical activity level spent in walking associated with comorbidity severity (Nechuta et al., 2013). So, breast cancer patients who reported lower levels of physical activity, specifically walking energy expenditure had comorbidities more often.

We assessed the quadriceps muscle strength for evaluating peripheral muscle strength and found that physical activity level and comorbidity level associated with quadriceps muscle strength. To our knowledge, during the treatment for breast cancer, metabolic changes like myotoxic response in the muscles can be affected with the implementation of cancer treatment (Visovsky, 2006). Cancer treatment may also damage the muscle itself (Loprinzi and Cardinal, 2012). These metabolic alterations finally decrease muscle force-generating capacity, leading to muscle weakness and functional decline. Increasing the physical activity level during and after the treatment may improve the muscle strength and control the atrophy.

In our study, no significant relationship found with functional exercise capacity and the physical activity level. The findings from breast cancer patients showed that lower functional capacity assessed with 6MWT distance does not show low physical activity. Explaining the mechanism as physical activity involved daily lifestyle whereas 6MWT is a clinical outcome. So we shouldn't estimate the physical activity level with aerobic exercise capacity and both measurements should be done together to assess functional impairment of the patients.

One out of five breast cancer survivors suffer from depression after oncology treatment, similar to other common cancer types (Khan and Amatya, 2013). Depressive symptoms were worse among more sedentary patients with breast cancer. This supported the idea of the controlling of depression oriented to improving functional status and muscle strength in the implementation of physical activity (Basen-Engquist et al., 2008; Ruland et al., 2013). Studies in general population showed that physically more active subjects are in a better mental health condition (Kahn et al., 2002). Counseling strategies could involve increasing physical activity through participation in support programs (Mutrie et al., 2012; Ruland et al., 2013).

Limitations: Some limitations of this study need to be discussed. The study involved relatively small sample size. There are other recognized potential variables affecting physical activity, such as sleep disturbances, pain, fatigue which were not included in this study and could affect the physical activity level. It needed to be addressed to compare the physical inactivity immediately after the oncological treatment with long term follow-up.

In conclusion, our study show that physical activity is independently related to peripheral muscle strength, comorbidity index, and psychosocial status in patients with breast cancer. Based on these findings, the influence of physical inactivity in peripheral muscle strength, comorbidity severity, and depression could help health professionals to improve adherence to physical activity programs in breast cancer patients.

\section{References}

Abu-Helalah M, Al-Hanaqta M, Alshraideh H, Abdulbaqi N, Hijazeen J (2014). Quality of life and psychological wellbeing of breast cancer survivors in Jordan. Asian Pac J Cancer Prev, 15, 5927-36.

American Thoracic Society (2002). ATS statement: guidelines for the six-minute walk test. Am J Respir Crit Care Med, 166, 111-7.

Ariza-Garcia A, Galiano-Castillo N, Cantarero-Villanueva I, et al (2013). Influence of physical inactivity in psychophysiolocigal state of breast cancer survivors. Eur $J$ Cancer Care, 22, 738-45.

Basen-Engquist K, Hughes D, Perkins H, et al (2008). Dimensions of physical activity and their relationship to physical and emotional symptoms in breast cancer survivors. J Cancer Surviv, 2, 253-61.

Bjelland I, Dahl AA, Haug TT, et al (2002). The validity of the hospital anxiety and depression scale. An updated literature review. J Psychosom Res, 52, 69-77.

Bleiker EM, Pouwer F, van der Ploeg HM, et al (2000). Psychological distress two years after diagnosis of breast cancer: frequency and prediction. Patient Educ Couns, 40, 
209-17.

Bohannon RW (2001). Measuring knee extensor muscle strength. Am J Phys Med Rehabil, 80, 13-8.

Braithwaite D, Moore DH, Satariano WA, et al (2012). Prognostic impact of comorbidity among long-term breast cancer survivors: results from the LACE study. Cancer Epidemiol Biomarkers Prev, 21, 1115-25.

Burnham TR, Wilcox A (2002). Effects of exercise on physiological and psychological variables in cancer survivors. Med Sci Sports Exerc, 34, 1863-7.

Cantarero-Villanueva I, Fernandez-Lao C, Fernandez DELPC, et al (2011). Associations among musculoskeletal impairments, depression, body image and fatigue in breast cancer survivors within the first year after treatment. Eur $J$ Cancer Care (Engl), 20, 632-9.

Charlier C, Pauwels E, Lechner L, et al (2012). Physical activity levels and supportive care needs for physical activity among breast cancer survivors with different psychosocial profiles: a cluster-analytical approach. Eur J Cancer Care, 21, 790-9.

Courneya KS, Friedenreich CM (2011). Physical activity and cancer: an introduction. Recent Results Cancer Res, 186, $1-10$.

Galanti G, Stefani L, Gensini G (2013). Exercise as a prescription therapy for breast and colon cancer survivors. Int J Gen Med, 6, 245-51.

Galiano-Castillo N, Ariza-Garcia A, Cantarero-Villanueva I, et al (2013). Depressed mood in breast cancer survivors: Associations with physical activity, cancer-related fatigue, quality of life, and fitness level. Eur J Oncol Nurs.

Jacobsen PB, Hann DM, Azzarello LM, et al (1999). Fatigue in women receiving adjuvant chemotherapy for breast cancer: characteristics, course, and correlates. J Pain Symptom Manage, 18, 233-42.

Kahn EB, Ramsey LT, Brownson RC, et al (2002). The effectiveness of interventions to increase physical activity. A systematic review. Am J Prev Med, 22, 73-107.

Khan F, Amatya B (2013). Factors associated with long-term functional outcomes, psychological sequelae and quality of life in persons after primary brain tumour. J Neurooncol, 111, 355-66.

Lee IM, Shiroma EJ, Lobelo F, et al (2012). Effect of physical inactivity on major non-communicable diseases worldwide: an analysis of burden of disease and life expectancy. Lancet, 380, 219-29.

Loprinzi PD, Cardinal BJ (2012). Effects of physical activity on common side effects of breast cancer treatment. Breast Cancer, 19, 4-10.

Lynch BM, Neilson HK, Friedenreich CM (2011). Physical activity and breast cancer prevention. Recent Results Cancer Res, 186, 13-42.

McTiernan A (2008). Mechanisms linking physical activity with cancer. Nat Rev Cancer, 8, 205-11.

Mohammadi S, Sulaiman S, Koon PB, Amani R, Hosseini SM (2013a). Impact of healthy eating practices and physical activity on quality of life among breast cancer survivors. Asian Pac J Cancer Prev, 14, 481-7.

Mohammadi S, Sulaiman S, Koon PB, Amani R, Hosseini SM (2013b). Association of nutritional status with quality of life in breast cancer survivors. Asian Pac J Cancer Prev, 14, 7749-55.

Mutrie N, Campbell A, Barry S, et al (2012). Five-year followup of participants in a randomised controlled trial showing benefits from exercise for breast cancer survivors during adjuvant treatment. Are there lasting effects? J Cancer Surviv, 6, 420-30.

Nechuta S, Lu W, Zheng Y, et al (2013). Comorbidities and breast cancer survival: a report from the shanghai breast cancer survival study. Breast Cancer Res Treat, 139, 227-35.

Parkin DM (2011). 9. Cancers attributable to inadequate physical exercise in the UK in 2010. Br J Cancer, 105, 38-41.

Ruland CM, Andersen T, Jeneson A, et al (2013). Effects of an internet support system to assist cancer patients in reducing symptom distress: a randomized controlled trial. Cancer Nurs, 36, 6-17.

Sabiston CM, Brunet J, Burke S (2012). Pain, movement, and mind: does physical activity mediate the relationship between pain and mental health among survivors of breast cancer? Clin J Pain, 28, 489-95.

Saglam M,Arikan H, Savci S, et al (2010). International physical activity questionnaire: reliability and validity of the Turkish version. Percept Mot Skills, 111, 278-84.

Schmitz KH, Courneya KS, Matthews C, et al (2010). American College of Sports Medicine roundtable on exercise guidelines for cancer survivors. Med Sci Sports Exerc, 42, 1409-26.

Turhal NS, Dane F, Sinav H, et al (2010). Anxiety and depression in Turkish breast cancer patients. J Buon, 15, 720-5.

Visovsky C (2006). Muscle strength, body composition, and physical activity in women receiving chemotherapy for breast cancer. Integr Cancer Ther, 5, 183-91.

Wang D, Zheng W, Wang SM, et al (2012). Estimation of cancer incidence and mortality attributable to overweight, obesity, and physical inactivity in China. Nutr Cancer, 64, 48-56.

Zainal NZ1, Nik-Jaafar NR, Baharudin A, Sabki ZA, Ng CG (2013). Impact of healthy eating practices and physical activity on quality of life among breast cancer survivors. Asian Pac J Cancer Prev, 14, 2649-56. 